\title{
Applications of Mollie Horadam's generalized integers to Fermatian and Fibonacci numbers
}

\author{
A. G. Shannon \\ Fellow, Warrane College, The University of New South Wales \\ Kensington NSW 2033, Australia \\ e-mails: tshannon38egmail.com, \\ t. shannon@warrane. unsw.edu. au
}

Received: 7 January 2019

Accepted: 11 March 2019

\begin{abstract}
This paper extends some of the arithmetic functions which Mollie Horadam developed for sequences of generalized integers and apply them to some particular integer sequences, particularly the Fibonacci and Fermatian numbers.
\end{abstract}

Keywords: Fermatian numbers, Fibonacci numbers, Lucas numbers, $q$-Bernoulli numbers, divisibility sequences, Ramanujan's sum, Möbius function, Totient functions, Co-prime.

2010 Mathematics Subject Classification: 11B75, 11Z05, 11B65.

\section{Introduction}

In a series of papers [9-16], the late Mollie Horadam, wife of Alwyn F. Horadam and mother of Kathie Horadam (another Professor of Mathematics in Australia), extended the classic arithmetic functions of number theory to generalizations of the integers. We shall further extend some of these function and consider Fermatian and Fibonacci numbers as examples of divisibility sequences $\left\{u_{n}\right\}$ with, for instance, $\theta\left(u_{n}\right), \varphi\left(u_{n}, u_{m}\right), \mu\left(u_{n}\right),\left(u_{n}, u_{m}\right)$ as analogues of ordinary arithmetical functions.

The approach of Mollie Horadam [16] and others, particularly David Daykin [6], was to start with generalized primes as a foundation for generalized integers. Here, though the approach is to start with divisibility sequences, the elements of which we consider as generalized integers, and to define some of their elements as generalized primes. That is, up is a generalized prime (modulo a divisibility sequence) if its only divisors are itself and unity (within the system).

Our generalized integers cannot necessarily be represented as a product of distinct generalized primes, as we shall see. Thus new analogues of the classical arithmetical functions 
are needed in order to study these divisibility sequences as generalizations of integers. This is not to say that we cannot redefine our generalized primes to fit Mollie Horadam's theory but it leads to unnecessarily complicated questions about the distribution of these generalized primes within a divisibility sequence.

\section{Fermatian numbers}

In a series of papers [9-16], the late Mollie Horadam, wife of Alwyn F. Horadam and mother of Kathie Horadam (another Professor of Mathematics in Australia), extended the classic

$$
\underline{z}_{n}=\left\{\begin{array}{cc}
-z^{n} \underline{z}_{n} & (n<0) \\
1+z+z^{2}+\ldots z^{n-1} & (n>0) \\
1 & (n=0)
\end{array}\right.
$$

so that

$$
\underline{1}_{n}=n \text {. }
$$

and

$$
\underline{1}_{n} !=n !,
$$

where

$$
\underline{z}_{n} !=\underline{z}_{n} \underline{z}_{n-1} \cdots_{z} \underline{z}_{1}
$$

For example, if we consider the Fermatian numbers of index 2, we have $\underline{2}_{2}=3, \underline{2}_{3}=7$, $\underline{2}_{4}=15, \underline{2}_{6}=63, \underline{2}_{8}=255$, so that $\underline{2}_{2}, \underline{2}_{3}$ and $\underline{2}_{4}$ are generalized Fermatian primes, and $\underline{2}_{6}=\left(\underline{2}_{2}\right)^{2} \underline{2}_{3}$, but $\underline{2}_{8}$ cannot be represented as a product of Fermatian numbers of index 2 . Some properties of these numbers may be found in [18] and Carlitz and Moser [4]. Carlitz has also used Fermatian numbers in the development of $q$-Bernoulli numbers and polynomials [2]. The first ten Fermatian numbers of the first ten indices are displayed in Table 1.

\begin{tabular}{|c|r|r|r|r|r|r|r|r|r|r|}
\hline $\begin{array}{c}\text { Index } \\
\Downarrow\end{array}$ & $\mathbf{1}$ & $\mathbf{2}$ & $\mathbf{3}$ & \multicolumn{1}{c|}{$\mathbf{4}$} & \multicolumn{1}{c|}{$\mathbf{5}$} & \multicolumn{1}{c}{$\mathbf{6}$} & $\mathbf{7}$ & $\mathbf{8}$ & $\mathbf{9}$ & $\mathbf{1 0}$ \\
\hline $\mathbf{1}$ & 1 & 2 & 3 & 4 & 5 & 6 & 7 & 8 & 9 & 10 \\
\hline $\mathbf{2}$ & 1 & 3 & 7 & 15 & 31 & 63 & 127 & 255 & 511 & 1023 \\
\hline $\mathbf{3}$ & 1 & 4 & 13 & 40 & 121 & 364 & 1093 & 3280 & 9841 & 29524 \\
\hline $\mathbf{4}$ & 1 & 5 & 21 & 85 & 341 & 1365 & 5461 & 21845 & 87381 & 349525 \\
\hline $\mathbf{5}$ & 1 & 6 & 31 & 156 & 781 & 3906 & 19531 & 97656 & 488281 & 2441406 \\
\hline $\mathbf{6}$ & 1 & 7 & 43 & 259 & 1555 & 9331 & 55987 & 335923 & 2015539 & 12093235 \\
\hline $\mathbf{7}$ & 1 & 8 & 57 & 400 & 2801 & 19608 & 137257 & 960800 & 6725601 & 47079208 \\
\hline $\mathbf{8}$ & 1 & 9 & 73 & 585 & 4681 & 37449 & 299593 & 2396745 & 19173961 & 153391689 \\
\hline $\mathbf{9}$ & 1 & 10 & 91 & 820 & 7381 & 66430 & 597871 & 5380840 & 48427561 & 435848050 \\
\hline $\mathbf{1 0}$ & 1 & 11 & 111 & 1111 & 11111 & 111111 & 1111111 & 11111111 & 111111111 & 1111111111 \\
\hline
\end{tabular}

Table 1. First 10 Fermatian numbers of the first 10 indices 
The corresponding row and column sequences, $\left\{z_{n}\right\}_{n=1}^{\infty},\left\{z_{n}\right\}_{z=1}^{\infty}$, are obvious from their construction, but the sequence, $\left\{\sum_{n=1}^{z-1}{\underline{(z-n)_{n}}}_{n}\right\} \equiv\{1,3,6,11,21,45,105,315,1058, \ldots\}$, formed from adding along the forward diagonals, does not seem to be well-known [20].

\section{Some arithmetical functions}

When dealing with ordinary integers we note that

$$
\delta(m, s)= \begin{cases}1, & m \mid s, \\ 0, & m / s,\end{cases}
$$

has different interpretations in Hardy and Wright [7] and Horadam [15], namely

$$
\begin{aligned}
\delta(m, s) & =g(s, m) / m \\
& =\left\{\begin{array}{r}
g(m) / m \\
E_{s}(m+1) .
\end{array}\right.
\end{aligned}
$$

With (3.1) we shall use

$$
\rho(n, s)=\left\{\begin{array}{lc}
0, & \text { if } \exists j: j \mid(n, s), 1<j<n, \\
1, & \text { otherwise, }
\end{array},\right.
$$

and $\mu(n)$, the Möbius multiplicative function, defined for all positive integers $n$ by

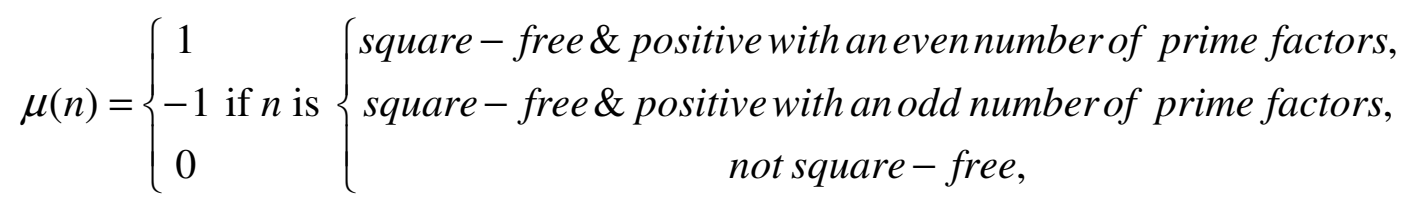

and $\mu(1)=1$. Examples of $\rho(n, s)$ are set out in Table 2 which illustrates that it can detect primes for numbers in this range:

\begin{tabular}{|c|c|c|c|c|c|c|c|c|}
\hline $\boldsymbol{n} \downarrow$ & $\mathbf{1}$ & $\mathbf{2}$ & $\mathbf{3}$ & $\mathbf{4}$ & $\mathbf{5}$ & $\mathbf{6}$ & $\mathbf{7}$ & $\mathbf{8}$ \\
\hline $\mathbf{1}$ & 1 & 1 & 1 & 1 & 1 & 1 & 1 & 1 \\
\hline $\mathbf{2}$ & 1 & 1 & 1 & 0 & 1 & 0 & 1 & 0 \\
\hline $\mathbf{3}$ & 1 & 1 & 1 & 1 & 1 & 0 & 1 & 1 \\
\hline $\mathbf{4}$ & 1 & 1 & 1 & 0 & 1 & 0 & 1 & 0 \\
\hline $\mathbf{5}$ & 1 & 1 & 1 & 1 & 1 & 1 & 1 & 1 \\
\hline $\mathbf{6}$ & 1 & 1 & 1 & 0 & 1 & 0 & 1 & 0 \\
\hline $\mathbf{7}$ & 1 & 1 & 1 & 1 & 1 & 1 & 1 & 1 \\
\hline $\mathbf{8}$ & 1 & 1 & 1 & 0 & 1 & 0 & 1 & 0 \\
\hline
\end{tabular}

Table 2. $\rho(n, s)$ for $n, s=1,2, \ldots, 8$ 
We shall also use

$$
\zeta_{m}=\exp (2 \pi i / m)
$$

We note that

$$
\begin{aligned}
\sum_{n \leq m} \zeta_{m}^{n s} & =g(s, m) \\
& =m \delta(m, s)
\end{aligned}
$$

and

$$
\sum_{\substack{n \leq m \\(n, m)=1}} \zeta_{m}^{n s}=c(s, m)
$$

Ramanujan's sum, which are proved in Hardy and Wright. Of marginal relevance to some of Mollie Horadam's work is the neat definition of generalized (order $r$ ) Lucas numbers by Williams [21] and a modification of Carlitz [3]:

$$
L_{s, n}^{(r)}=\frac{1}{d} \sum_{j=1}^{r} \alpha_{r, j}^{n} \zeta_{r}^{s(j-1)}, s=0,1,2, \ldots, r-1,
$$

in which $d$ is some real number for $r>2$. When $r=2, d=\alpha_{2,1}-\alpha_{2,2}$, and

$$
L_{0, n}^{(2)}=\alpha_{2,1}^{n}+\alpha_{2,2}^{n}
$$

the primordial numbers of Lucas [17].

\section{Some generalised integer divisors}

For notational convenience with generalized integers $\left\{u_{n}\right\}$, we let

$$
\delta\left(u_{m}, u_{s}\right)=\delta\{m, s\}=\left\{\begin{array}{cc}
1 & u_{m} \mid u_{s}, \\
0 & u_{m} \mid u_{s},
\end{array}\right.
$$

and

$$
\rho\left(u_{m}, u_{s}\right)=\rho\{m, s\}=\left\{\begin{array}{cc}
0 & \text { if } \exists u_{j}: u_{j} \mid u_{s}, u_{1}<u_{j}<u_{m}, \\
1 & \text { otherwise. }
\end{array}\right.
$$

For $u_{d}, u_{j}, u_{k} \in\left\{u_{n}\right\}$, we call $u_{d}=\left(u_{j}, u_{k}\right)$ the greatest common divisor of $u_{j}$ and $u_{k}$, if

a) $\quad u_{d} \mid u_{j}$ and $u_{d} \mid u_{k}$,

b) every $u_{c} \mid u_{d}$ if $u_{c} \mid u_{j}$ and $u_{c} \mid u_{k}, u_{c} \in\left\{u_{n}\right\}$.

When $u_{1}=\left(u_{j}, u_{k}\right)$, shall say that $u_{j}$ and $u_{k}$ are generalized co-primes. A formula for the greatest common divisor is then given by

$$
\left(u_{s}, u_{t}\right)=\max \left\{u_{m} \delta\{m, s\}, \delta\{m, t\}\right\}, 1 \leq m \leq \min \{s, t\} \text {, for each } u_{m} \mid \min \{s, t\} .
$$

For example, for the Fibonacci numbers: $\left(F_{4}, F_{8}\right)=(3,21)=\max \{1,2,4\}=3$, and also for the Fermatian numbers: $\left(\underline{3}_{4}, \underline{3}_{8}\right)=(40,3280)=\max \{1,2,4\}=40$. 
Proof of (4.3). For each $\left\{\left(u_{m} \mid u_{s}\right) \wedge\left(u_{m} \mid u_{t}\right)\right\}, 1 \leq m \leq \min \{s, t\}, u_{m}=u_{m} \delta(m, s) \delta(m, s)$, and so the maximum of all these will be the greatest common divisor of $u_{s}$ and $u_{t}$.

We now assert that $\rho\{m, s\} \delta\{m, s\}=1$ iff $u_{m}$ is unity or the smallest prime factor of $u_{s}$. Proof. If $u_{m}$ is the smallest prime factor of $u_{s}$, then $\delta\{m, s\}=1$ because $u_{m} \mid u_{s}$, and $\rho\{m, s\}=1$ because $\nexists u_{j} \mid u_{s}: 1<u_{j}<u_{m}$. Also $\rho\{1, s\} \delta\{1, s\} \forall s$.

If $\rho\{m, s\} \delta\{m, s\} \neq 1$, then $\rho\{m, s\} \delta\{m, s\}=0$, and either

(a) $\rho\{m, s\}=0$, which implies that $\exists u_{j}: 1<u_{j}<u_{m}, u_{j} \mid u_{s}$, or

(b) $\delta\{m, s\}=0$, which implies that $u_{m} \wedge u_{s}$,

or both (a) and (b), all of which imply that $u_{m}$ is not unity or is not the smallest prime divisor.

For example, for the Fermatian numbers when $q=2$,

$$
\begin{aligned}
& \rho\{2,6\}=\rho\{3,63\}=1, \\
& \delta\{2,6\}=\delta\{3,63\}=1,
\end{aligned}
$$

so

$$
\rho\{2,6\}=\delta\{2,6\}=1 ;
$$

but $\rho\{3,6\}=\rho\{7,63\}=0$, since $1<3<7$ and 3 । 63 , so

$$
\rho\{3,6\} \delta\{3,6\}=0 .
$$

Also

$$
\rho\{7,7,\}=\rho\{127,127\}=1,
$$

since $\nexists \underline{2}_{n}: 1<n<7: \underline{2}_{n} \mid 127$, and so

$$
\rho\{7,7\} \delta\{7,7\}=1,
$$

but

$$
\delta\{3,7\}=\delta\{7,127\}=0,
$$

so

$$
\rho\{3,7\} \delta\{3,7\}=0 .
$$

\section{Sums of generalized divisors}

Let

$$
\sigma_{k}\left(u_{n}\right)=\sum_{u_{d} \mid u_{n}} u_{d}^{k}
$$

so that $\sigma_{0}\left(u_{n}\right)$ represents the number of divisors $u_{d}$ of $u_{n}$, where $u_{d}, u_{n} \in\left\{u_{n}\right\}$. We can also define a generalization of the greatest integer function, $\left[u_{s} / u_{m}\right]$, by the recurrence relation:

$$
\left[u_{s} / u_{m}\right]-\left[u_{s-1} / u_{m}\right]=\delta\{m, s\},\left[u_{0} / u_{m}\right]=0 .
$$


Thus, when $\left\{u_{n}\right\}=\{n\},\left[u_{s} / u_{m}\right]=[s / m]$, the ordinary greatest integer function.

Then, analogous to the well-known

$$
\sum_{m=1}^{n}[n / m]=\sum_{s=1}^{n} \sigma_{0}(s)
$$

we have

$$
\sum_{m=1}^{n}\left[u_{n} / u_{m}\right]=\sum_{s=1}^{n} \sigma_{0}\left(u_{s}\right) \text {, if }\left\lfloor u_{j-1} / u_{j}\right\rfloor=0 \forall j
$$

Proof.

$$
\begin{aligned}
\sigma_{0}\left(u_{s}\right) & =\sum_{m=1}^{s} \delta\{m, s\} \\
& =\sum_{m=1}^{s}\left(\left[u_{s} / u_{m}\right]-\left[u_{s-1} / u_{m}\right]\right) . \\
\sum_{s=1}^{n} \sigma_{0}\left(u_{s}\right) & =\sum_{m=1}^{n}\left(\sum_{s=m}^{n}\left[u_{s} / u_{m}\right]-\left[u_{s-1} / u_{m}\right]\right) \\
& =\sum_{m=1}^{n}\left(\sum_{s=m}^{n}\left[\frac{u_{s}}{u_{m}}\right]-\sum_{s=m-1}^{n-1}\left[\frac{u_{s}}{u_{m}}\right]\right) \\
& =\sum_{m=1}^{n}\left(\left[\frac{u_{n}}{u_{m}}\right]-\left[\frac{u_{m-1}}{u_{m}}\right]\right) \\
& =\sum_{m=1}^{n}\left[\frac{u_{n}}{u_{m}}\right] .
\end{aligned}
$$

Note that

$$
\left[\frac{u_{s}}{u_{m}}\right]=\sum_{j=1}^{s} \delta\{m, j\}
$$

Proof.

$$
\begin{gathered}
{\left[\frac{u_{s}}{u_{m}}\right]-\left[\frac{u_{s-1}}{u_{m}}\right]=\delta\{m, s\}} \\
{\left[\frac{u_{s-1}}{u_{m}}\right]-\left[\frac{u_{s-2}}{u_{m}}\right]=\delta\{m, s-1\}} \\
{\left[\frac{u_{1}}{u_{m}}\right]-\left[\frac{u_{0}}{u_{m}}\right]=\delta\{m, 1\} .}
\end{gathered}
$$

On adding the corresponding sides of these equations we get the result we seek, since $\left[u_{0} / u_{m}\right]=0$. 
A corollary of this is that $\left[u_{s}\right]=\sum_{j=1}^{s} \delta\{1, j\}$, which is the number of the elements of the sequence which are less than or equal to $u_{s}$. We can then obtain that

$$
\left[\frac{u_{s}}{u_{m}}\right]-\left[\frac{u_{s-1}}{u_{m}}\right]=\int_{m}^{m+1} \delta\{\lfloor\rfloor, s\} d y .
$$

Proof.

$$
\begin{aligned}
\int_{m}^{m+1} \delta\{\lfloor y\rfloor, s\} d y & =\left\{\begin{array}{cc}
\int_{m}^{m+1} d y, & u_{m} \mid u_{s}, \\
0, & u_{m} \mid u_{s},
\end{array}\right. \\
& = \begin{cases}1, & u_{m} \mid u_{s}, \\
0, & u_{m} \mid u_{s},\end{cases} \\
& =\delta\{m, s\} \\
& =\left[\frac{u_{s}}{u_{m}}\right]-\left[\frac{u_{s-1}}{u_{m}}\right] .
\end{aligned}
$$

For ordinary integers this becomes

$$
\left.\left\lfloor\frac{s}{m}\right\rfloor-\left\lfloor\frac{s-1}{m}\right\rfloor=\int_{m}^{m+1} \delta\{y\rfloor, s\right\} d y .
$$

Thus,

$$
\sigma_{0}\left(u_{n}\right)=\int_{n}^{n+1} \int_{1}^{n+1} \delta\{\lfloor y\rfloor,\lfloor x\rfloor\} d y d x
$$

Proof.

$$
\begin{aligned}
\sigma_{0}\left(u_{n}\right) & =\sum_{u_{d} \mid u_{n}} 1 \\
& =\sum_{i=1}^{n} \delta\{i, n\} \\
& =\sum_{i=1}^{n} \int_{i}^{i+1} \delta\{\lfloor y\rfloor, n\} \\
& =\int_{n}^{n+1} \int_{1}^{n+1} \delta\{\lfloor y\rfloor,\lfloor x\rfloor\} d y d x .
\end{aligned}
$$

For example, for the sequence of Fibonacci numbers, since the divisors of $F_{4}=3$, are $F_{1}, F_{2}$ and $F_{4}, \sigma_{0}\left(F_{4}\right)=3$, and

$$
\begin{aligned}
\int_{4}^{5} \int_{1}^{5} \delta\{\lfloor y\rfloor,\lfloor x\rfloor\} d y d x & =\int_{4}^{5}\left(\int_{1}^{3} d y+\int_{4}^{5} d y\right) d x \\
& =3 \int_{4}^{5} d x \\
& =3 .
\end{aligned}
$$


For ordinary integers, the result (5.6) becomes similar to a result of Graeme Cohen, formerly Editor of the Bulletin of the Australian Mathematical Society, namely

$$
\sigma_{0}(n)=\int_{n}^{n+1} \int_{1}^{n+1} \delta(\lfloor y\rfloor,\lfloor x\rfloor) d y d x .
$$

Cohen [5] has also considered aspects of Gaussian integers as generalized integers.

\section{Prime divisors}

Let $w\left\{u_{n}\right\}$ denote the number of prime divisors of $u_{n}$ as in [9]. For notational convenience, let $w\{1\}=1$. Then

$$
w\{n\}=\sum_{1<u_{i}<u_{n}} \rho\{i, i\} \delta\{i, n\}
$$

Proof. Let

$$
u_{n}=\prod_{j=1}^{s} u_{p_{j}}^{a_{g} N}
$$

in which $(N=1) \vee\left(N \notin\left\{u_{n}\right\}\right)$. Then

$$
\begin{aligned}
\sum_{1<u_{i}<u_{n}} \rho\{i, i\} \delta\{i, n\} & =\sum_{j=1}^{n-1} \rho(p, p) \delta\{j, n\} \\
& =w\{n\}
\end{aligned}
$$

where $p \equiv u_{p j}^{a_{j}}$, because $\rho\{p, p\}=0$ except when $a_{j}=1$, since $u_{p_{j}} \mid u_{p_{j}}^{a_{j}}, u_{1}<u_{p_{j}}<u_{p_{j}}^{a_{j}}$ for $a_{j}>1$.

Examples follow:

(i) For the ordinary integers when $n=12$ :

$$
\begin{aligned}
w(12)= & \sum_{i=2}^{11} \rho\{i, i\} \delta\{i, 12\} \\
= & \rho(2,2) \delta(2,12)+\rho(3,3) \delta(3,12)+ \\
& \rho(4,4) \delta(4,12)+\rho(6,6) \delta(6,12) \\
= & 2
\end{aligned}
$$

Since in Table $1, \rho(2,2)=\rho(3,3)=1, \rho(4,4)=\rho(6,6)=0$.

(ii) For the Fibonacci numbers, where $F_{8}=21=7 F_{4}$, and

$$
\begin{aligned}
w\{8\} & =\sum_{1<F_{1}<F_{8}} \rho\{i, i\} \delta\{i, 8\} \\
& =\rho\{4,4\} \delta\{4,8\} \\
& =1 .
\end{aligned}
$$

We note that we can express $w\{n\}$ similarly to (5.6) as

$$
w\{n\}=\int_{n}^{n+1} \int_{2}^{n+1} \rho\{j, j\} \delta\{[y\rfloor,\lfloor x\rfloor\} d y d x .
$$

We now define an analogue of the Möbius function 


$$
\mu\left(u_{n}\right)=\left\{\begin{array}{cc}
0, & u_{p}^{2} \mid u_{n}, \\
(-1)^{w\{n\}}, & \text { otherwise. }
\end{array}\right.
$$

For example, for the Fibonacci numbers:

$$
\mu\left(F_{3}\right)=\mu(2)=(-1)^{1}=1, \mu\left(F_{1}\right)=\mu(1)=(-1)^{0}=1 .
$$

Then

$$
\mu\left(u_{n}\right)=(-1)^{w\{n\}}\left(1-\delta\left(u_{p}^{2}, u_{n}\right)\right)
$$

Proof: When $n=1, u_{n}=1$, and

$$
\mu\left(u_{1}\right)=(-1)^{w\{1\}}\left(1-\delta\left(u_{p}^{2}, u_{1}\right)\right)
$$

When $n=p>1, u_{n}=u_{p}$, and

$$
\begin{aligned}
\mu\left(u_{p}\right) & =(-1)^{w\left(u_{p}\right)}\left(1-\delta\left(u_{p}^{2}, u_{p}\right)\right) \\
& =-1 . \\
u_{n} & =u_{p}^{a} N, a>1, \delta\left(u_{p}^{2}, u_{n}\right)=1
\end{aligned}
$$

and

$$
\mu\left(u_{n}\right)=0 .
$$

If $\left(u_{m}, u_{n}\right)=1$, then $\mu\left(u_{n}\right)$ is multiplicative, that is,

$$
\mu\left(u_{n}\right) \mu\left(u_{m}\right)=\mu\left(u_{n} u_{m}\right)
$$

Proof: If $u_{m}$ and $u_{n}$ have no common divisors in the sequence $\left\{u_{n}\right\}$, then

$$
w\{m n\}=w\{m\}+w\{n\} .
$$

Analogous to the Mőbius function theorem we have

$$
\sum_{\substack{u_{d} u_{c}=u_{n} \\
\left(u_{d}, u_{c}\right)=1}} \mu\left(u_{d}\right)=\left\{\begin{array}{ll}
1, & n=1, \\
0, & n>1,
\end{array}\right\}=e(n) .
$$

Proof: If $n=1$, then

$$
\begin{aligned}
\mu\left(u_{d}\right) & =\mu\left(u_{1}\right) \\
& =(-1)^{0} \\
& =1 .
\end{aligned}
$$

If $n>1$, then

$$
\begin{aligned}
\mu\left(u_{n}\right) & =\mu\left(\prod_{i=1}^{s} u_{p_{i}}^{a_{i}}\right) \\
& =\prod_{i=1}^{s} \mu\left(u_{p_{i}}^{a_{i}}\right) \\
\sum_{\substack{u_{d} u_{c}=u_{p_{a}}^{a_{i}} \\
\left(u_{d}, u_{c}\right)=1}} \mu\left(u_{d}\right) & =\mu\left(u_{1}\right)+\sum \mu\left(u_{p_{i}}^{a_{i}}\right) \\
& =1+(-1)^{1} \\
& =0 .
\end{aligned}
$$


In the same spirit we now define $\varphi\left(u_{n}\right)$ to be the number of elements of the set $\left\{u_{1}, u_{2}, \ldots, u_{n-1}\right\}$ which are co-prime with $u_{n}$. For instance, if $\left\{u_{n}\right\}=\{1,2,3,1,2,3, \ldots\}$, then the co-primes of $u_{5}$ are $u_{1}=1, u_{3}=3, u_{3}=3, u_{4}=1$, and so $\varphi\left(u_{5}\right)=3$. We are then able to assert the following theorem:

$$
\varphi\left(u_{n}\right)=n-1-\psi\left(u_{n}\right)
$$

in which

$$
\psi\left(u_{n}\right)=\sum_{\substack{m=2 \\ u_{m} \neq 1}}^{n-1} \sum_{s=2}^{n-1} \delta\{m, s\} \delta\{m, n\} \rho\{m, s\} .
$$

Proof. Let $A=\left\{u_{1}, u_{2}, \ldots, u_{n}\right\}$. Then for each $m$

- $\sum_{m=1}^{n-1} \sum_{s=1}^{n-1} \delta\{m, s\} \delta\{m, n\}$ represents the number of elements $u_{s}, u_{1} \leq u_{s}<u_{n}:\left(u_{s}, u_{n}\right)=u_{m} \geq 1$;

- $\sum_{\substack{m=2 \\ u_{m} \neq 1}}^{n-1} \sum_{s=2}^{n-1} \delta\{m, s\} \delta\{m, n\}$ represents the number of elements $u_{s}, u_{1} \leq u_{s}<u_{n}:\left(u_{s}, u_{n}\right)=u_{m}>1$;

- $\sum_{\substack{m=2 \\ u_{m} \neq 1}}^{n-1} \sum_{s=2}^{n-1} \delta\{m, s\} \delta\{m, n\} \rho\{m, s\}$ : number of distinct elements $u_{s}, u_{1} \leq u_{s}<u_{n}:\left(u_{s}, u_{n}\right)=u_{m}>1$.

Thus $n-1$ represents the number of distinct elements of $A$ which are co-prime with $u_{n}$, and this is $\varphi\left(u_{n}\right)$; that is,

$$
\varphi\left(u_{n}\right)=n-1-\psi\left(u_{n}\right)
$$

Observe that $\psi\left(u_{p}\right)=0$ only when $u_{p}$ is a generalized prime. More generally, we note that the problem of Lehmer, discussed by Alter [1], of whether there exists a composite integer $n$ and integer $k>1$ so that the equation

$$
k \varphi(n)=n-1
$$

has a solution can be made equivalent to finding whether there is a solution to

$$
j \varphi(n)=\psi(n)
$$

for a composite integer $n$ and a positive integer $j$.

Examples of the use of (6.7) now follow in (i), (ii) and (iii) below.

(i) For $\left\{u_{n}\right\}=\{1,2,3,1,2,3, \ldots\}, u_{1}=1, u_{2}=2, u_{3}=3, u_{4}=1, u_{5}=2$, and 1, 1, 3, 1 are co-prime with 2 , so that 


$$
\begin{aligned}
\varphi\left(u_{5}\right) & =3 \\
\psi\left(u_{5}\right) & =\sum_{\substack{m=2 \\
u_{m} \neq 1}}^{4} \sum_{s=2}^{4} \rho\{m, s\} \delta\{m, s\} \delta\{m, 5\} \\
& =\sum_{s=2}^{4} \rho\{2, s\} \delta\{2, s\} \\
& =\rho\{2,2\} \\
& =1
\end{aligned}
$$

that is,

$$
\begin{aligned}
\varphi\left(u_{5}\right) & =n-1-\psi\left(u_{5}\right) \\
& =5-1-1 .
\end{aligned}
$$

(ii) For $\left\{F_{n}\right\}, F_{1}=1, F_{2}=1, F_{4}=3, F_{5}=5$ are co-prime with $F_{6}=8$, so that

$$
\begin{aligned}
\varphi\left(F_{6}\right) & =4 \\
\psi\left(F_{6}\right) & =\sum_{\substack{m=2 \\
F_{m} \neq 1}}^{5} \sum_{s=2}^{5} \rho\{m, s\} \delta\{m, s\} \delta\{m, 6\} \\
& =\sum_{s=2}^{5} \rho\{3, s\} \delta\{3, s\} \\
& =\rho\{3,3\} \\
& =1
\end{aligned}
$$

so that

$$
\begin{aligned}
\varphi\left(F_{6}\right) & =n-1-\psi\left(F_{6}\right) \\
& =6-1-1 .
\end{aligned}
$$

(iii) For $Z, 1,5,7,11$ are co-prime with 12 , so that

$$
\begin{aligned}
\varphi(12)= & 4 \\
\psi(12)= & \sum_{m=2}^{11} \sum_{s=2}^{11} \rho(m, s) \delta(m, s) \delta(m, 12) \\
= & \rho(2,2) \delta(2,2)+\rho(2,4) \delta(2,4)+\rho(2,6) \delta(2,6)+ \\
& \rho(2,8) \delta(2,8)+\rho(2,10) \delta(2,10)+ \\
& \rho(3,3) \delta(3,3)+\rho(3,9) \delta(3,9) \\
= & 7, \\
\varphi(12)= & 12-1-\psi(12) \\
= & 4 .
\end{aligned}
$$

A consequence of this is that for $u_{p} \neq 1$ if $p>2$ :

$$
\varphi\left(u_{p}\right)=p-1,
$$

Since

$$
\delta\{m, p\}=0, m>1 .
$$

Furthermore, if $u_{p} \neq 1$ if $p>2$, then $u_{p}$ is a generalized prime iff $p$ is an ordinary prime. 
Proof: $u_{n} \mid u_{p}$ iff $n \mid p$ iff $n=1, p\left(u_{p} \neq 1\right.$ if $\left.p>2\right)$, and thus $u_{n} \mid u_{p}$ iff $u_{n}=u_{1}=1$ or $u_{n}=u_{p}$, which is a result used in a proof of a generalized Staudt-Clausen theorem [8].

\section{Concluding comments}

Analogues of other functions can be similarly defined; for example, the Nagell totient function for elements of a divisibility sequence. We define $\theta\left(u_{n}, u_{t}\right)$ as the number of elements $u_{j}$ of the set $\left\{u_{1}, u_{2}, \ldots, u_{t}\right\}, t \leq n$, such that

$$
\left(u_{n-j}, u_{t}\right)=\left(u_{j}, u_{t}\right)=1 \text {. }
$$

We then have

$$
\theta\left(u_{n}, u_{t}\right)=\sum_{j=1}^{t} \delta\left(\left(u_{n-j}, u_{t}\right), u_{1}\right) \delta\left(\left(u_{j}, u_{t}\right), u_{1}\right)
$$

Proof. The two delta functions in the summation are both unity only when $\left(u_{n-j}, u_{t}\right) \mid u_{1}$ and when $\left(u_{j}, u_{t}\right) \mid u_{1}$; that is, only when

$$
\left(u_{n-j}, u_{t}\right)=\left(u_{j}, u_{t}\right)=1 .
$$

When we sum over all $j$ up to and including $t$ we have the number of elements which satisfy the conditions of Nagell's function for divisibility sequences.

As a corollary we have

$$
\begin{aligned}
\varphi\left(u_{n}\right) & =\theta\left(u_{n}, u_{n}\right) \\
& =\sum_{j=1}^{n} \delta\left(\left(u_{n-j}, u_{n}\right), u_{1}\right) \delta\left(\left(u_{j}, u_{n}, u_{1}\right)\right)
\end{aligned}
$$

since this yields the number of elements of the set $\left\{u_{1}, u_{2}, \ldots, u_{n-1}\right\}$ which are coprime with $u_{n}$.

We can also define $\pi\left(u_{n}\right)$ to represent the number of generalized primes $\leq u_{n}$. Then

$$
\pi\left(u_{n}\right)=\sum_{m=2}^{n} \rho\{m, m\}
$$

Proof. For $m \geq 2$,

$$
\rho\{m, m\}=\left\{\begin{array}{rr}
0, & \text { if } u_{m} \text { has a factorbetween } u_{1} \text { and } u_{m} \\
1, & \text { if } u_{m} \text { is a generalized prime. }
\end{array}\right.
$$

For example,

$$
\begin{aligned}
\pi\left(F_{8}\right) & =\sum_{m=2}^{8} \rho\{m, m\} \\
& =5 \\
& \neq \pi(8) .
\end{aligned}
$$


Of course there are still unsolved problems with some of the classical functions in terms of the ordinary integers [22]. A further development of a calculus of convolutions is unnecessary as this has been achieved by Dr Mollie Horadam; what has been done here is to outline some properties of the $\rho, \delta$ functions which are pertinent to the study of divisibility sequences.

\section{References}

[1] Alter, R. (1973). Can $\varphi(n)$ properly divide $n$ - 1? American Mathematical Monthly. 80, 192-193.

[2] Carlitz, L. (1948). q-Bernoulli numbers and polynomials. Duke Mathematical Journal. $15,987-1000$.

[3] Carlitz, L. (1960). Some arithmetic sums connected with the greatest integer function. Mathematica Scandinavica. 8, 59-64.

[4] Carlitz, L., L. Moser. (1966). On some special factorizations of $(1-x n) /(1-x)$. Canadian Mathematical Bulletin. 9, 421-426.

[5] Cohen, G. L. (1975). Selberg formulae for Gaussian integers. Acta Arithmetica. 16, $385-400$.

[6] Daykin, D. E. (1963). Linear sequences over a finite field. American Mathematical Monthly. 70, 637-642.

[7] Hardy, G.H., E.M. Wright. (1965). An Introduction to the Theory of Numbers. 4th edition. Oxford: Clarendon Press.

[8] Horadam, A.F., A.G. Shannon. (1976). Ward's Staudt-Clausen problem. Mathematica Scandinavica. 29 (4), 239-250.

[9] Horadam, E. M. (1961). Arithmetical functions of generalized primes. American Mathematical Monthly. 68, 626-629.

[10] Horadam, E. M. (1962). Arithmetical functions associated with the unitary divisors of a generalized integer. American Mathematical Monthly. 69, 196-199.

[11] Horadam, E. M. (1963). The Euler $\varphi$ function for generalized integers. Proceedings of the American Mathematical Society. 14, 754-772.

[12] Horadam, E. M. (1963). A calculus of convolutions for generalized integers. Indagationes Mathematicae. 25, 695-698.

[13] Horadam, E. M. (1964). Ramanujan's sum for generalized integers. Duke Mathematical Journal. 31, 697-702.

[14] Horadam, E. M. (1966). Addendum to Ramanujan's sum for generalized integers. Duke Mathematical Journal. 33, 705-707.

[15] Horadam, E. M. (1966). Exponential functions for arithmetical semi-groups. Journal für die reine und angewandte Mathematik. 222, 14-19. 
[16] Horadam, E. M. (1971). An extension of Daykin's generalized Möbius function to unitary divisors. Journal für die reine und angewandte Mathematik. 246, 117-125.

[17] Lucas, E. (1878). Théorie des fonctions numériques simplement périodiques. American Journal of Mathematics. 1: 184-240; 289-321.

[18] Shannon, A. G. (2004). Some properties of Fermatian numbers. Notes on Number Theory \& Discrete Mathematics, 10 (2), 25-33.

[19] Shannon, A.G., J.V. Leyendekkers. (2014). Some properties associated with a prime characteristic function. Proceedings of the Jangjeon Mathematical Society. 17 (2), 293-297.

[20] Sloane, N.J.A., Simon Ploufffe. (1995). The Encyclopedia of Integer Sequences. San Diego, CA: Academic Press; Available online: https://oise.org/.

[21] Williams, H. C. (1972). On a generalization of the Lucas functions. Acta Arithmetica. 20, $33-51$.

[22] Wooldridge, Kent. (1979). Values taken many times by Euler's phi-function. Proceedings of the American Mathematical Society. 76 (2), 229-234. 